\title{
Astroparticle Physics at Eastern Colombia
}

\author{
Hernán Asorey \\ Laboratorio Detección de Partículas y Radiación, \\ Centro Atómico Bariloche e Instituto Balseiro (CNEA/UNCuyo) \\ San Carlos de Bariloche, Río Negro-Argentina and \\ Escuela de Física - Universidad Industrial de Santander \\ Bucaramanga, Santander-Colombia. \\ Luis A. Núñez \\ Escuela de Física - Universidad Industrial de Santander \\ Bucaramanga, Santander-Colombia and \\ Departamento de Fúsica, Universidad de Los Andes, Mérida-Venezuela.
}

June 24, 2021

\begin{abstract}
We present the emerging panorama of Astroparticle Physics at Eastern Colombia, and describe several ongoing projects, most of them related to the Latin American Giant Observatory (LAGO) Project. This research work is carried out at the Grupo de Investigaciones en Relatividad y Gravitación of Universidad Industrial de Santander.
\end{abstract}

\section{INTRODUCTION}

Astroparticle Physics is now one of the most exciting interdisciplinary fields in High Energy Physics, where particle Physics and Astrophysics share satellites and ground based detectors to explore new phenomena and innovative applications.

In Colombia, since 2010, at the Universidad Industrial de Santander (UIS, Bucaramanga), the Relativity and Gravitation Research Group (GIRG for its Spanish acronym for Grupo de Investigación en Relatividad y Gravitación) develops a research line in close contact with researchers of the Latin American Giant Observatory (LAGO, formerly known as Large Aperture GRB Observatory) project. 


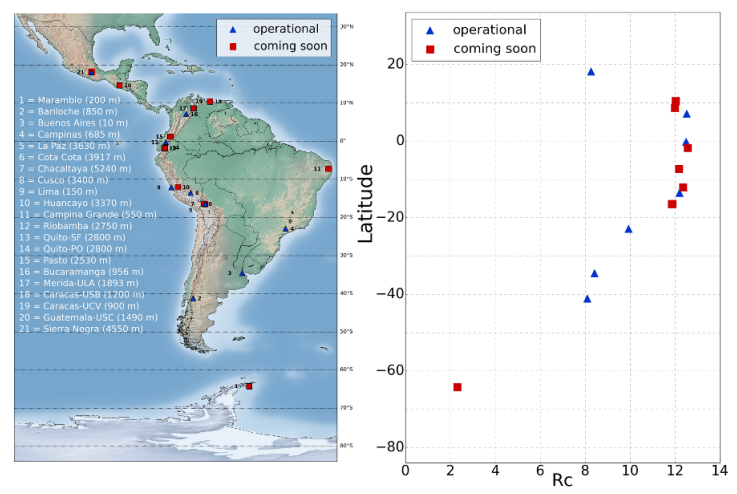

Figure 1: The Sites of the Latin American Giant Observatory (LAGO), currently located in eight countries at Latin America. The first detectors are in operation (blue triangles), and some other detectors (red squares), are planned to start in 2015-2016 [1, 5]. In the right panel, the vertical rigidity cut-off of each site, calculated according the procedure described in section 3 and [4, are shown.

The Latin American Giant Observatory [1] is a highly integrated and collaborative research and academic project with more than eighty Ibero American astroparticle researchers, motivated by the experience of the Pierre Auger Observatory [2] in Argentina, and devoted to study space weather effects [3] and high energy transient phenomena such as Gamma Ray Bursts (GRB) on ground-based detectors [4]. Long-term modulation and transient events can also be characterized by using the LAGO detection network, as it spans over a big area with different sites at different latitudes, longitudes and geomagnetic rigidity cut-offs. Presently LAGO has 10 country members (Argentina, Bolivia, Brazil, Colombia, Ecuador, Guatemala, Mexico, Peru, Spain and Venezuela) spanning a non-centralized and collaborative network of 25 institutions with 10 ground-based Water Cherenkov Detector (WCDs) [5], located at different altitudes from Mexico through Patagonia. Other detectors are expected to be up and running in the near future including two WCD in the Antarctica Peninsula [6], see Figure 1.

This work is organised as follows: in the next section we describe two new analysis of the scalers rates (an implementation of the single particle technique [7]) of the LAGO WCDs installed at Mount Chacaltaya. One of these analysis is conducted to search short transient events ( $\Delta t \lesssim 1$ minute) and the other is aimed to find periodic long term signals. Next, we present the 
LAGO Space Weather program and show how flux variations of secondary particles at ground level can be related to heliospheric and geomagnetic modulation of Galactic Cosmic Rays and provide precise information of the variable conditions of the near-Earth space environment. With this toolkit, developed to study the Space Weather phenomena, we are able to easily determine the total integrated flux of cosmic radiation in any place at the Earth surface or even, e.g., along specifics commercial flight trajectories. For this particular case, we calculate the integrated exposure due to the radiation background during a flight and its modulation by effects such as altitude, latitude, exposure time and transient space weather events. Following the description of our projects, we prove the capabilities of single WCD to detect transient space weather phenomena from ground level. The introduction of the Multi-Spectral Analysis Technique also shows that using even a single detector it is possible to determine the flux at bands that are dominated by different types of particles coming from different primaries contributions.

\section{SEARCHING FOR SHORT/LONG TERM SIG- NALS IN LAGO}

In this section we present a selection of our observations which confirm that, when using an adapted analysis technique to the characteristics of our small detectors, it is possible to observe, from the ground level and in the LAGO network of WCDs, different type of signals of different astrophysics nature at different time scales.

\subsection{Short Term Signals: Searching for Gamma Ray Burst}

LAGO site in Bolivia is located at Mount Chacaltaya $\left(16^{\circ} 21^{\prime} 00^{\prime \prime} \mathrm{S}, 68^{\circ} 07^{\prime} 53^{\prime \prime} \mathrm{W}\right)$, at $5270 \mathrm{~m}$ above sea level, where the instrumented area and the high altitude of the site provide enough sensitivity to detect high energy Gamma Ray Bursts (GRBs) [8]. Recent studies [9], based on CORSIKA simulations [10], show that the angular aperture of the Chacaltaya site can be extended up to a zenith angle of $25^{\circ}$ in the energy range of interest for GRB. Combining these result with the uptime of each of the three WCD at Chacaltaya in this period, the total exposure of this site accumulated during the 2010-2012 period was $2.7 \times 10^{8} \mathrm{~m}^{2} \mathrm{~s}$ sr [4].

Several validations of data quality cuts were introduced in all datasets collected [11] and, by using the moving window average (MWA) method 
[12], we look for $\geq|3 \sigma|$ deviations in the central $5 \mathrm{~ms}$ bin on a 2 minutes $(24,000$ time bins). If such a deviation is observed in at least two operating detectors and is present in both the low and the intermediate deposited energy sub-channels, the signal is tagged as a potential transient (GRB) candidate.

Applying this approach over 2 terabytes of data collected in the period 2010-2012 at Chacaltaya, we found a potential candidate, started on Wed Dic 07 15:45:49.675 \pm 0.005 UTC 2011 (unix time (1323272749.675 \pm 0.005 ) s). At this time, the equatorial coordinates of the zenith at Chacaltaya were RA/Dec (J2000) $16^{\mathrm{h}} 17^{\mathrm{m}} 31.3^{\mathrm{s}} /-16^{\circ} 21^{\prime} 00^{\prime}$, with an acceptance aperture of $\theta \lesssim 25^{\circ}$. After a careful examination of the signals shapes, calibration data and operation metadata of our detectors and the atmospheric database of this site, we discarded the possibility that this event was produced by detectors malfunctions, HF noises, electric lightnings or other phenomena of atmospheric origin.

The detected signals are shown in figures 2, We did not find any similar events registered on both the SWIFT [13] and Fermi satellites [14] and also at the Gamma-ray Coordinates Network (GCN) database ${ }^{1}$. However, at this time the field of view of Fermi was well outside of our acceptance cone in Chacaltaya [4].

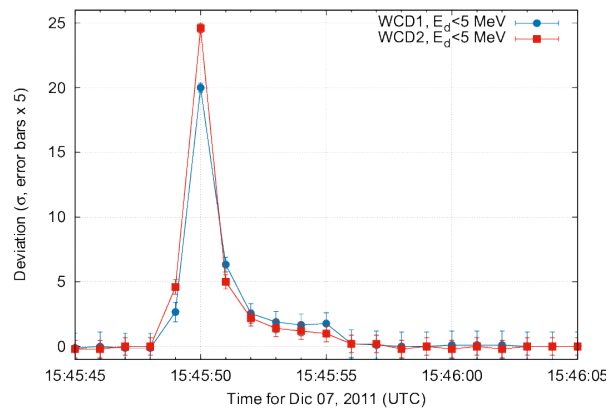

(a)

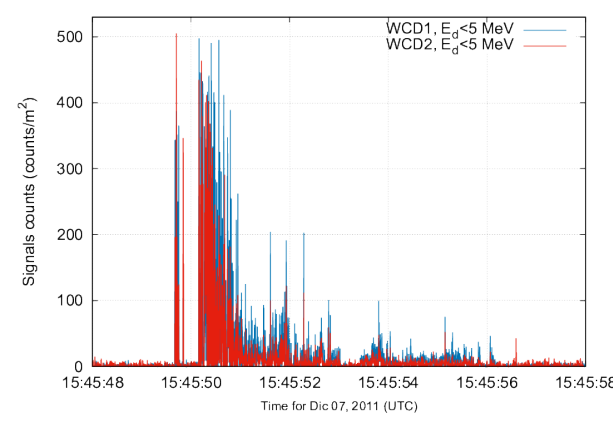

(b)

Figure 2: Potential candidate signals for the event registered on Wed Dic 07 15:47:02.378 UTC 2011. In panel (a) we show our alert as described in the text, while in the panel (b) the registered signals in $5 \mathrm{~ms}$ temporal bins are shown

\footnotetext{
${ }^{1}$ http://gcn.gsfc.nasa.gov/
} 


\subsection{Lont Term Signals: Epoch Analysis}

On the search for periodic signals we performed a series of analyses over large periods of time on the data collected at the Chacaltaya station. Data stacking or summation in two different time systems, solar and sidereal, were made over the data. The idea behind this process is based on the random, poissonian, nature of the majority of the radiation measured by our detectors. If any not random, periodic, signal were to exist, and if sufficient data were to be summed in a scale in which this signal happened at the same time every day or month or week, an indication of the underlying signal would appear. No matter how small this signal could be it will build an observable rise on the line that represent the summed data, as the random fluctuations will eventually cancel and the signal will grow 9 . This summation of data were made on two different systems: sidereal time, for signals coming from outside of our Solar System and in solar time, for signals modulated by Solar activity. A one-minute average signals were summed with the average of the corresponding minute of the next day, solar or sidereal.

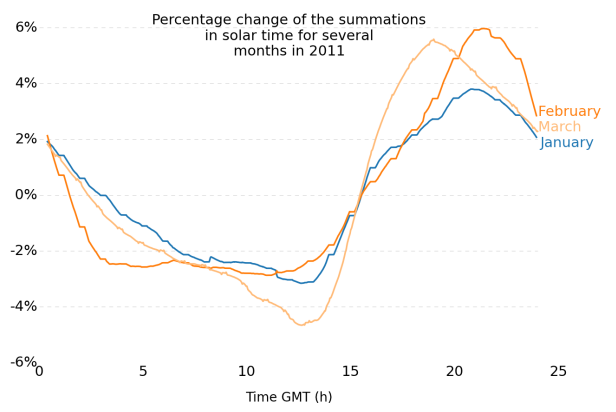

(a)

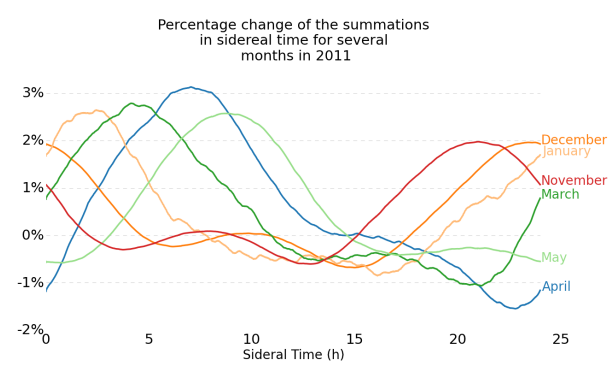

(b)

Figure 3: Solar daily modulation in the flux of cosmic rays observed at the LAGO site in Chacaltaya. This phenomena was observed by stacking and summing the measured and corrected fluxes in solar (a) and sidereal (b) times.

The results of the complete summation on the data collected in the Chacaltaya station in 2011 is show in Fig. 3 where both solar time and sidereal times are shown here, and have consistent results. In solar time it appears a rise on the counts that holds in the same stage of the solar day while in sidereal time the rise moves about two hour every month. These facts are clear indicators of the solar nature of this phenomenon. The amplitude and phase observed on the solar time variations are consistent 
with the well known daily modulation of solar origin in the flux of low energy cosmic rays. This result also shown that our single WCD, combined with the appropriated data acquisition and analysis techniques, are capable to detect space weather related phenomena from the Earth surface.

\section{THE LAGO SPACE WEATHER PROGRAM}

The LAGO Space Weather (LAGO-SW) program studies how flux variations of secondary particles at ground level can be related to heliospheric modulation of GCRs and provides precise information of variable conditions of the near-Earth space environment [15, 3]. It is supported by an intensive and detailed chain of simulations, accounting three important factors: the geomagnetic effects, the development of the extensive air showers (EAS) in the atmosphere, and the detector response to the different types of secondary particles at ground level by means of a GEANT4 model [16, 17, 3].

\subsection{Geomagnetic Field Effects}

The geomagnetic field (GF) effects on the propagation of charged particles that could contribute to the background radiation at ground level can be characterized by the directional rigidity cut-off $R_{c}$ at each LAGO site (see figure 1), considered as a function of the geographical position at some altitude (Lat, Lon, Alt) and the arrival direction $(\theta, \phi)$ of the primary at this point. Additionally, a time stamp (TS YYMMDDHHMMSS UTC) should also be included to address transient phenomena during those periods of intense geomagnetic activity, so $R_{c} \equiv R_{c}$ (TS, Lat, Lon, Alt, $\left.\theta, \phi\right)$.

This directional $R_{c}$ can be calculated at each LAGO site using the Magnetocosmics code [18] by applying the backtracking technique [19]. We use the International Geomagnetic Field Reference (IGRF) version 11 [20] for modeling the near-earth GF $\left(r<5 R_{\oplus}\right)$ and the Tsyganenkov (TSY01) [21] to describe the outer GF. As it is commonly used, our description of the GF can be tagged by six parameters: solar wind dynamic pressure, $\mathrm{D}_{\mathrm{ST}}$ index, $B_{y}$ and $B_{z}$ components of the geomagnetic field (in GSM system), and the $G_{1}$ and $G_{2}$ parameters of TSY01 model. 


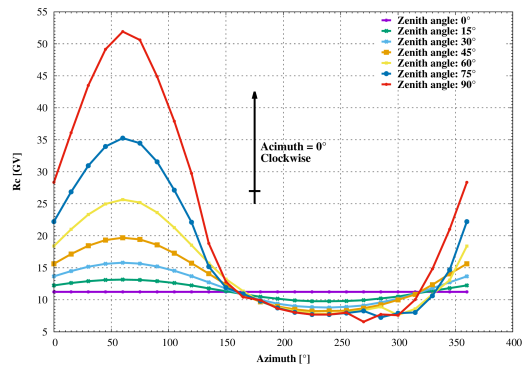

(a)

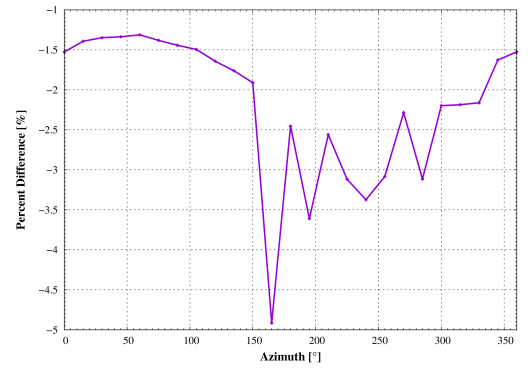

(b)

Figure 4: (a) Directional rigidity cut-off $\left(R_{c}\right)$ at the atmosphere edge, as a function of the azimuth angle $\phi$ and for different zenith angles $\theta$ for the LAGO site in Bucamaranga, Colombia, during secular conditions of the geomagnetic field. The effect of the SAA is observed for inclined particles in the range $250^{\circ}<\phi<300^{\circ}$. (b) Relative difference for $\theta=45^{\circ}$ between the $R_{c}$ under secular condition (left) and during the geomagnetic storm occurred on May 15th 2005 09:00 UTC.

\subsection{Secondary Particles Flux at Ground Level}

The second set of simulation correspond to CORSIKA 2 (author?) [10] of the secondaries observed at ground level by the EAS produced during the interaction with the atmosphere of the complete flux of primaries. The flux of all nuclei primaries in the range $1 \leq Z \leq 26$ was assumed to be uniform in solid angle and considering a single power law for each nuclei to describe the energy dependence in the range $Z \times R_{c} \leq E \leq 1 \mathrm{PeV}$ [22, 23, 15, 3]. The corresponding parameters were obtained from [24]. As the $R_{c}$ depends on the arrival direction of each primary, the total number of primaries $N$ is strongly angular dependent.

The effect of the geomagnetic field in the flux of primaries impinging the Earth atmosphere can be observed in Fig. 5(a), where a comparison of fluxes with/without GF before the edge of the atmosphere is shown. Only primaries that actually produced secondary particles at ground are considered. Low energy nuclei are affected, but it is not a sharp cutoff as the studied effect depends on its $Z$, on the arrival directions of the primaries, and also includes the effect of atmospheric absorption of secondary particles during the EAS development.

Figure 5(b) is a comparison of the expected flux without corrections

\footnotetext{
${ }^{2}$ v7.3500 with QGSJET-II-04; GHEISHA-2002; EGS4; Curve, External Atmosphere, Volumetric Detector
} 


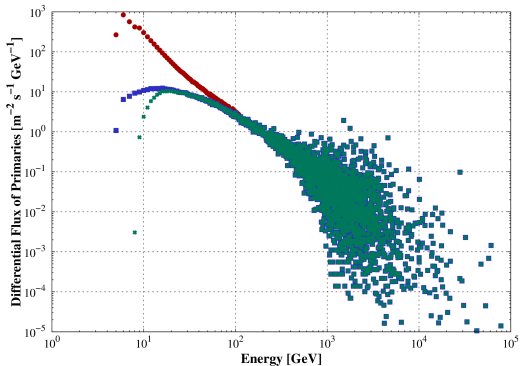

(a)

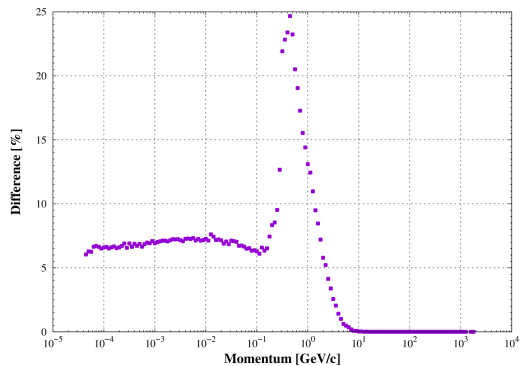

(b)

Figure 5: (a) Geomagnetic effects in the flux of low energy GCR, observed when comparing the flux of primaries that actually produced secondary particles at ground when GF corrections for secular conditions are included (green stars) and when they are not considered (blue squares). The total flux of injected primaries is also included (red circles). (b) The GF effect in the secondary particles at ground level as a function of the secondary momentum. In this curve, the relative difference between the flux without GF corrections and the corrected flux is shown. The larger differences are observed at $p_{\mathrm{sec}} \simeq 500 \mathrm{MeV} / \mathrm{c}$. In this region, the flux of secondaries is dominated by neutrons.

with the corresponding geomagnetically corrected one and relative difference between these two cases is shown as a function of the particle momentum. The observed differences are large enough to justify the incorporation of geomagnetic corrections when low energy secondary flux is calculated. A large deviation in the total flux is also observed, which is peaked at $p_{\mathrm{sec}} \simeq$ $500 \mathrm{MeV} / \mathrm{c}$. At this values, the secondary flux is dominated by neutrons. For this particular component, the decrease at ground level due to GF effect in secular conditions represents a diminution of $-36.6 \%$. This could be an indication of the sensitivity of secondary neutron flux as a proxy of the changing conditions in the near-earth space environment.

\subsection{Multi-spectral Analysis of Space Weather Phenomena}

The charge histogram of the WCD, typically used for detector calibration (see for example [25]), is obtained by time integration of the individual pulses measured in the WCD. The resulting histogram, showed in figure 6(a), is originated by the convolution of the response of the WCD to the different types of EAS particles and the water quality, the inner coating used, the PMT size and the detector geometry. By doing first principle calculations 
and detailed simulations, it is well established that the small signal region, corresponding to low values of deposited energy in the detector volume, $E_{d}$, is dominated by the electromagnetic component (EM, $\gamma_{\mathrm{s}}$ and $e^{ \pm}$) of the shower; while high values of $E_{d}$ correspond to the simultaneous entrance (within the electronic sampling time, $10 \mathrm{~ns}-40 \mathrm{~ns}$ ) of multiple particles to the detector volume (the so called mini-shower - MS - regime). Finally, intermediate values of $E_{d}$, evidenced by a characteristic peak 3 in the histogram called the muon hump, are dominated by single muons through the detector. By using these histogram features we are able to determine the flux of secondary particles at different bands of deposited energy in the detector by using pulse shape discrimination techniques. This is what we called the multi-spectral analysis technique (MSAT).

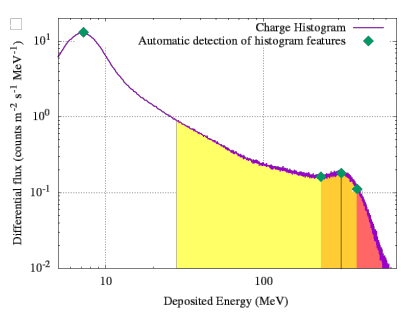

(a)

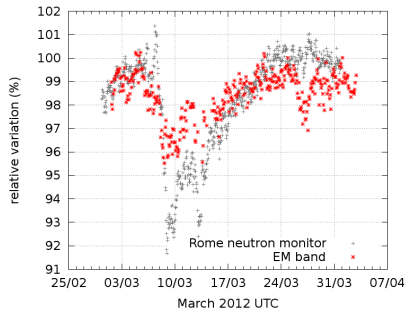

(b)

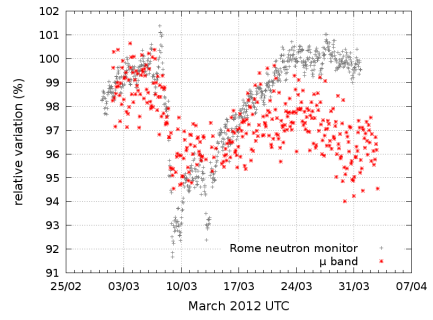

(c)

Figure 6: (a) Charge histogram of one LAGO WCD in Bariloche, Argentina. A fully automated algorithm look for histogram features (green diamonds) to define integrations bands (shaded regions). Each integration band is dominated by different type of particles: EM particles (yellow), muons (orange) and multiple particles (pink). Plates (b) and (c) display results of Multi-Spectal Analysis of the Forbush Decrease of March 8th, 2012 measured in a single $1.8 \mathrm{~m}^{2}$ WCD installed in Bariloche, Argentina (red stars), compared with measurements of the Rome neutron monitor (gray pluses): Electromagnetic-band (a), and $\mu$-band (b).

We applied the MSAT to analyze the Forbush event associated with the passage of an interplanetary coronal mass ejection detected on March 8th, 2012, by different instruments [26]. The studied data were acquired in a single $1.8 \mathrm{~m}^{2}$ WCD (with $2.5 \mathrm{~m}^{3}$ of pure water and a 8 " Hamamatsu R5912 PMT) at the LAGO site of Bariloche, Argentina. After applied flux pressure correction to each band, the Forbush decrease is clearly visible in our MSAT,

\footnotetext{
${ }^{3}$ The first peak seen at the histogram at very low signals is originated by the detector trigger system and is not considered in these analysis.
} 
with a maximum peak-to-peak decrease of $\sim 5 \%, \sim 6 \%$, and $\sim 4 \%$ in the EM, $\mu$ and MS bands respectively. The temporal evolution of the EM and $\mu$ bands are shown in Fig. 6(b) and 6(c), where, respectively, our data is compared with the one of Rome neutron monitor 4 . Solar daily modulation of the flux is also visible on both bands. The particular features observed in our data are currently under careful analysis and will be published soon.

\subsection{Cosmic Rays Induced Background Radiation On Board of Commercial Flights}

In this subsection we apply the techniques described before to determine the total integrated flux of cosmic radiation which a commercial aircraft is exposed to along specific flight trajectories [27]. To study the radiation background during a flight and its modulation by effects such as altitude, latitude, exposure time and transient magnetospheric events, we perform simulations based on Magnetocosmics and CORSIKA codes.

As before, the expected flux of secondary particles in any place along the plane trajectory is based on the simulation of the complete flux of cosmic rays primaries within a given range of energy, that includes the effect of the rigidity cut-off at different locations in the Earth, that we summarize here:

1. Simulation of showers at different altitudes using CORSIKA. Features of injected primaries at the top of the atmosphere:

- Primary nuclei injected: $1 \leq Z_{p} \leq 26,1 \leq A_{p} \leq 56$

- Very low initial rigidity cut-off rigidity: $R_{c}=4 G V$

- Energy and arrival direction: $\left(R_{c} \times Z_{p}\right) \leq\left(E_{p} / \mathrm{GeV}\right) \leq 10^{6}$, $0^{\circ} \leq \theta_{p} \leq 90^{\circ}, 0^{\circ} \leq \phi_{p} \leq 360^{\circ}$

- Simulation time: $t=7200 \mathrm{~s}$ (primary particles flux is constant and isotropic)

2. Selection and discretization of routes.

3. Computation of rigidity cut-offs for each point in the trajectory using Magnetocosmics.

4. Filter secondary particles by the primary rigidities and the rigidity cut-off computed for each point of the trajectory: all those showers generated by primary particles with rigidities below the cut-off are simply discarded.

\footnotetext{
${ }^{4}$ Available at the NEST repository, http://www.nmdb.eu/nest
} 
5. Computation of the total amount of particles that hit the aircraft, by point-to-point integration of the flux of secondaries along the flight trajectory.

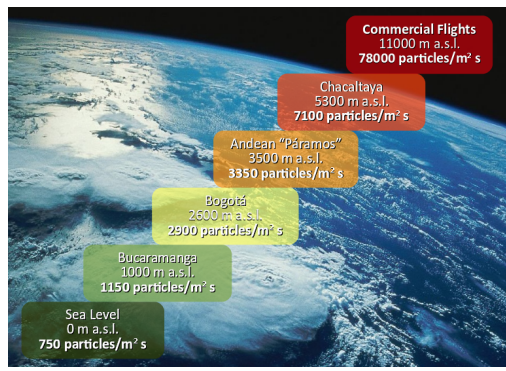

(a)

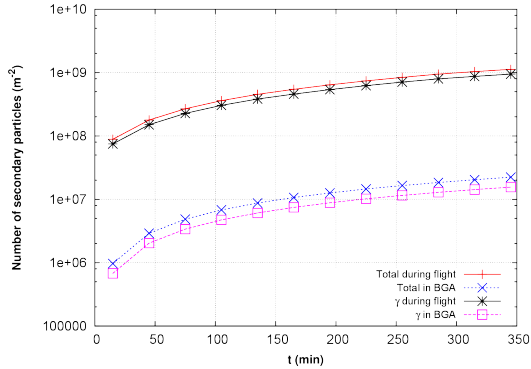

(b)

Figure 7: (a) Total particle flux as function of the altitude at different places on Earth. The atmospheric effect is large enough at high altitudes to justify a detailed simulation at flight level. (b) Integrated flux of particles (red plus signs) and only photons (black asterisks) as a function of time for the flight BOG-EZE, without taken into account the fuselage shield and the effects produced by takeoff and landing. As a comparison, we show the total integrated flux (blue crosses) and photons (magenta squares) for the same calculation but staying the same time at the city of Bucaramanga (Colombia), at an altitude of $1 \mathrm{~km}$ a.s.l. There is up to two orders of magnitude in the integrated exposure between those two considered cases.

As a first estimation, we chose the trajectory of the flight AR1360 BOGEZE (Bogotá-Buenos Aires), and can be seen elsewher"5. This route was divided into 12 intervals of equal flight time $(\sim 30$ minutes each $)$ and the flux of secondaries along each of them was assumed to be constant and equal to the flux in the midpoint. The shield due to the flight fuselage and the effects of takeoff and landing on the flux was not included in this preliminary analysis (i.e., the aircraft was supposed to fly at a constant altitude of $11 \mathrm{~km}$ along the whole trajectory). For each one of this intermediate points, the geomagnetic rigidity cut-off was calculated by using the method described in the previous section. The result of this calculation can be seen in figure 7 , where we show the integrated flux of total particles and photons as a function of time expected on board of a commercial flight. As a comparison, in the same figure we show the integrated flux expected for the same calculation

\footnotetext{
${ }^{5}$ See for example http://www.flightradar24.com/data/flights/ar1360/
} 
but staying for the same time at the city of Bucaramanga (Colombia) at an altitude of $1000 \mathrm{~m}$ a.s.l. In the same figure the effect of the atmospheric absorption on the EM component is clearly visible as a diminish of the photon flux at Bucaramanga when compared with the total flux respect to the diminution at $11 \mathrm{~km}$ a.s.l. A significant difference in the integrated flux of secondary particles have been obtained when compared typical flight level altitudes with ground level. Further investigations are been carried out to include several other factors, such as the fuselage effect and the conversion to equivalent doses in living tissues by using, e.g., Geant4 anthropomorphic phantoms (see for example [28]).

\section{DATA ACCESSIBILITY, REPRODUCIBILITY AND TRUSTWORTHINESS WITH LAGO DATA REPOSITORY}

The geographic distribution of our detectors (see figure 1), some of them located at very remote sites, introduce outstanding challenges for the reliable transference of high volumes of data. Moreover, this data is acquired on WCD of different geometries and characteristics. In this section we shall describe the ecosystem of data tools and services that we had been implemented to help solving the Data Accessibility, Reproducibility and Trustworthiness (DART) challenge in LAGO [29].

The DART initiative was launched by CHAIN-REDS (Coordination and Harmonisation of Advanced e-infrastructure for Research and Education Data Sharing): an European Commission co-project focused on promoting and supporting technological and scientific collaboration across different communities in various continents [30, 31]. This initiative provided a set of interrelated tools and services, based on worldwide adopted standards, to provide easy/seamless access datasets, data/documents repositories and the applications that could generate and/or make use of them.

Trustworthiness can be associated to data curation, particularly on the quality of the metadata describing the experimental protocol and data provenance [32, while reproducibility and replicability are closely connected to the accessibility to data sources and the possibility to manipulate/analyze data contained in them 33, 34].

CHAIN-REDS approach to data trustworthiness and reproducibility is based on the cornerstones:

\footnotetext{
${ }^{6}$ http://www.chain-project.eu
} 
1. adoption of standards for data discoverability, provenance and recoverability.

2. enablement of datasets authorships and user authentication with the corresponding assignments of specific roles on data services, which can be implemented by two strategies: assignment of Persistent Identifiers (PIDs) 35] to name data in a unique and timeless manner, ensuring that future changes on URIs or internal organization of databases will be transparent to the user; and implementation of a federated identity provision, a secure, flexible and portable mechanism to access e-infrastructures worldwide, based on agreements and standards [30].

3. access to a plethora of computing power to analyze the retrieved data or to contrast them to simulations through an intuitive web-interface.

\section{THE PAS PROJECT: ASTROPARTICLE PHYSICS AT THE COLOMBIAN PARAMO}

Motivated by all these developments, and aimed by the international interest to develop a near equatorial site devoted for the detection of high and ultra high energy cosmic rays [36], we started the development of the PAS (Social Astronomical Pole, Polo de Astronomía Social) [37]. It has two main objectives: to build a World class centre in astroparticle physics and related sciences, and at the same time, to become a permanent link between Science and Society.

Due to its geographic characteristics, the Andean Páramo located near Berlín, Colombia $(+7.13 \mathrm{~N},-72.9 \mathrm{~W}, 3450 \mathrm{~m}$ a.s.l. $)$, is an excellent location to build an array of particle detectors to study cosmic rays in a wide energy range, including solar activity modulation of cosmic rays, gamma astronomy, and the high energy region of the cosmic rays spectrum. Moreover, it can be easily extended to the highest energy region.

The design of the detector array is based on CORSIKA and GEANT4based detector response simulations, and deeply supported by the design experiences of previous arrays of WCD, such as the Pierre Auger Observatory. As the number of computational resources rapidly increases with the primary energy, several statistical techniques have been developed to simplify those calculations. It is common to implement the so called thinning algorithms [38] to reduce the number of secondary particles by assigning weights to representative particles in the evolution of the cascade. However, since this is a compression method with loss of information, it is required to 
recover the original flux of secondary particles without introducing artificial biasses. The so called de-thinning method [39] is one of the existent methods designed to deal with this information loss. To validate the first calculations at the Cosmic Ray knee energy level and beyond, we developed our own implementation, python based, of the de-thinning method, while introducing some physical improvements to the original method, such as the inclusion of local atmospheric models or secondary particle dependant atmospheric interaction lengths [40]. By using this method, we were able to decrease the computational resources to accomplish the first round of calculations needed to verify our first principle calculations for the design of the PAS array.

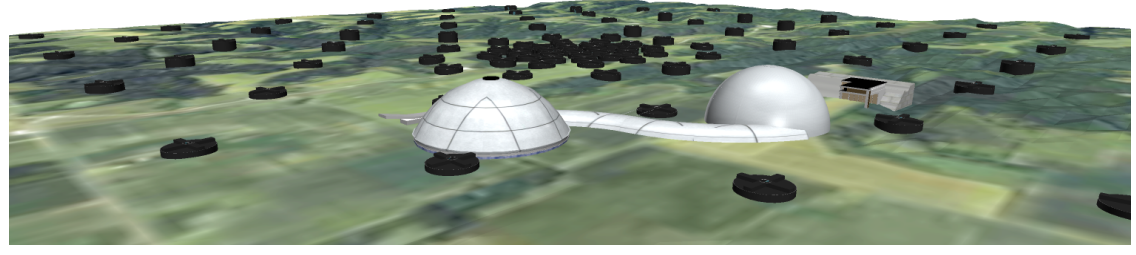

Figure 8: The PAS project concept: the "Science dome", the "Society dome" and the "tunnel of Science" establishing a bridge, a link, between Science and Society. An aerial view of the PAS array is also shown: 125 fully automated and autonomous WCD arranged in $\mathrm{a} \sim 16 \mathrm{~km}^{2}$ triangular graded grid.

The proposed design of this array, consisting in 125 fully automated and autonomous WCD arranged in a triangular graded grid and covering a total area of $\sim 16 \mathrm{~km}^{2}$, will allow to implement two different measurement modes: the counting, LAGO-like, mode and the shower mode. In the counting mode, the several techniques described before are used to study the flux variation of particles at detector level. In the shower mode, in contrast, we will look for time-space correlated signals in different detectors of the array. In this way, it will be possible to determine the main parameters that characterize the extensive air shower (EAS) produced by the interaction of a single high-energy cosmic ray with the atmosphere. The size of the array and the increasing spacing between detectors will allow to complement present measurements in the so called knee region of the cosmic ray energy spectrum $\left(E \sim 10^{15} \mathrm{eV}\right)$ and beyond.

Our second objective for PAS will be reached by the installation of three buildings: one $12 \mathrm{~m}$ hemispherical dome ("Society"), will harbor a Convention and Data Visualization Center and a Digital Planetarium; the second dome ("Science"), will hosts labs and offices for all the scientific activities, 
including a 20 inches optical fully automatized telescope for outreach activities and astronomical research at the Paramos. A first sketch of the design of this facilities is shown in Figure 8. The third building is the one that complete our concept, where an interactive visualization wall and roof will reproduce different animations and simulations on different science topics. This is the "Science tunnel", which will be the place where a true link between science and society shall be established.

\section{ACKNOWLEDGMENTS}

We thank the LAGO Collaboration and Vicerrectoría de Investigación y Extensión (UIS) for their permanent support. We also thanks to the Vicerrectoría Académica (UIS) and to ELCIRA (Europe Latin America Collaborative e-Infrastructure for Research Activities) Project for funding.

\section{References}

[1] H. Asorey, S. Dasso, and the LAGO Collaboration. LAGO: the latin american giant observatory. In The 34th International Cosmic Ray Conference, volume PoS(ICRC2015), page 247, 2015.

[2] The Pierre Auger Collaboration. The Pierre Auger Cosmic Ray Observatory. Submitted to Nucl. Inst. Meth. A., page arXiv:1502.01323., 2015.

[3] H. Asorey, S. Dasso, L.A. Núñez, Y. Pérez, C. Sarmiento-Cano, M. Suárez-Durán, and for the LAGO Collaboration. The LAGO space weather program: Directional geomagnetic effects, background fluence calculations and multi-spectral data analysis. In The 34th International Cosmic Ray Conference, volume PoS(ICRC2015), page 142, 2015.

[4] H. Asorey, P. Miranda, A. Núñez-Castiñeyra, L.A. Núñez, J. Salinas, C. Sarmiento-Cano, R. Ticona, A. Velarde, and the LAGO Collaboration. Analysis of background cosmic ray rate in the 2010-2012 period from the LAGO detectors at chacaltaya. In The 34th International Cosmic Ray Conference, volume PoS(ICRC2015), page 414, 2015.

[5] I. Sidelnik and the LAGO Collaboration. The sites of the latin american giant observatory. In The 34th International Cosmic Ray Conference, volume PoS(ICRC2015), page 665, 2015. 
[6] S. Dasso, A.M. Gulisano, J.J. Masías-Meza, H. Asorey, and the LAGO Collaboration. A project to install water-cherenkov detectors in the antarctic peninsula as part of the LAGO detection network. In The 34 th International Cosmic Ray Conference, volume PoS(ICRC2015), page 105, 2015.

[7] S. Vernetto. Detection of gamma-ray bursts in the $1 \mathrm{GeV}-1 \mathrm{TeV}$ energy range by ground-based experiments. Astroparticle Physics, 13:75-86, March 2000.

[8] D. Allard, I. Allekotte, C. Alvarez, H. Asorey, H. Barros, X. Bertou, O. Burgoa, M. Gómez Berisso, O. Martínez, and P. Miranda Loza. Use of water-cherenkov detectors to detect gamma ray bursts at the large aperture grb observatory (lago). Nuclear Inst. and Methods in Physics Research, A, 595(1):70-72, 2008.

[9] A. Nuñez-Castiñeyra. Sensibilidad del proyecto lago a señales gamma proveniente del centro de la galaxia. Tesis pregrado, Departamento de Física, Facultad de Ciencias, Universidad de lo Andes, Venezuela, May 2015.

[10] D. Heck, J. Knapp, J.N. Capdevielle, G. Schatz, and T. Thouw. Corsika : A monte carlo code to simulate extensive air showers. Technical Report FZKA 6019, Forschungszentrum Karlsruhe GmbH, 1998.

[11] L.A. Núñez, F. Quiñonez, and C. Sarmiento-Cano. Validación del linaje de los datos de la colaboración lago. instalaciones sierra negra y chacaltaya. ITECKNE, 10(1):104-112, 2013.

[12] C. Sarmiento-Cano. Identificación de destellos gamma en los repositorios de datos de la colaboración lago. Tesis pregrado, Escuela de Física, Universidad Industrial de Santander, Bucaramanga - Colombia, 2012.

[13] S. D. Barthelmy, L. M. Barbier, J. R. Cummings, E. E. Fenimore, N. Gehrels, D. Hullinger, H. A. Krimm, C. B. Markwardt, D. M. Palmer, A. Parsons, G. Sato, M. Suzuki, T. Takahashi, M. Tashiro, and J. Tueller. The burst alert telescope (bat) on the swift midex mission. Space Science Reviews, 120:143-164, October 2005.

[14] F. Acero, M. Ackermann, M. Ajello, A. Albert, W. B. Atwood, M. Axelsson, L. Baldini, J. Ballet, et al. Fermi large area telescope third source catalog. The Astrophysical Journal Supplement Series, 218(2):23, 2015. 
[15] The LAGO Collaboration [H. Asorey]. The LAGO Solar Project. In Proceedings of the 33th International Cosmic Ray Conference ICRC 2013, Río de Janeiro, Brazil, 2013.

[16] R. Calderón, H. Asorey, L. A. Núñez, and LAGO Collaboration. Geant4 based simulation of the water cherenkov detectors of the lago project. arXiv preprint, page arXiv:1503.07270, 2015.

[17] L. Otiniano, F. Quispe, J. Truyenque, S. Vargas, and the LAGO Collaboration. Development of a high altitude lago site in peru. In The 34 th International Cosmic Ray Conference, volume PoS(ICRC2015), page 688, 2015.

[18] L. Desorgher. MAGNETOSCOSMICS, Geant4 application for simulating the propagation of cosmic rays through the Earth magnetosphere, 2003.

[19] J. J. Masías-Meza and S. Dasso. Geomagnetic effects on cosmic ray propagation under different conditions for buenos aires and marambio, argentina. Sun and Geosphere, 9:41-47, 2014.

[20] International Association of Geomagnetism Aeronomy Working Group V-MOD. International geomagnetic reference field: the eleventh generation. Geophysical Journal International, 183(3):1216-1230, 2010.

[21] N. A. Tsyganenko. A model of the near magnetosphere with a dawndusk asymmetry 1. mathematical structure. Journal of Geophysical Research: Space Physics, 107(A8):SMP 12-1-SMP 12-15, 2002.

[22] H. Asorey. Measurement of Low Energy Cosmic Radiation with the Water Cherenkov Detector Array of the Pierre Auger Observatory. In Proceedings of the 32th International Cosmic Ray Conference ICRC 2011, volume 11, pages 462-465, Beijing, China, 2011. Chinese Academy of Sciences.

[23] S. Dasso and H. Asorey. The scaler mode in the Pierre Auger Observatory to study heliospheric modulation of cosmic rays. Advances in Space Research, 49(11):1563-1569, January 2012.

[24] P.K.F. Grieder. Cosmic rays at Earth, volume 1. Elsevier B.V., 2001.

[25] X. Bertou, P.S. Allison, C. Bonifazi, P. Bauleo, C.M. Grunfeld, M. Aglietta, F. Arneodo, D. Barnhill, J.J. Beatty, N.G. Busca, A. Creusot, 
D. Dornic, A. Etchegoyen, A. Filevitch, P.L. Ghia, I. Lhenry-Yvon, M.C. Medina, E. Moreno, D. Nitz, T. Ohnuki, S. Ranchon, H. Salazar, T. Suomijarvi, D. Supanitsky, A. Tripathi, M. Urban, and L. Villasenor. Calibration of the surface array of the pierre auger observatory. Nuclear Instruments and Methods in Physics Research Section A: Accelerators, Spectrometers, Detectors and Associated Equipment, 568(2):839 - 846, 2006.

[26] V. Alekseenko, F. Arneodo, G. Bruno, W. Fulgione, D. Gromushkin, O. Shchegolev, Yu Stenkin, V. Stepanov, and V. Sulakov. Registration of forbush decrease 2012/03/08 with a global net of the thermal neutron scintillation en -detectors. Journal of Physics: Conference Series, 409(1):012190, 2013.

[27] S. Pinilla, H. Asorey, and L.A. Núñez. Cosmic rays induced background radiation on board of commercial flights. arXiv preprint, page arXiv:1503.06193, 2015.

[28] D. Matthiä, T. Berger, and G. Reitz. Organ shielding and doses in lowearth orbit calculated for spherical and anthropomorphic phantoms. Advances in Space Research, 52(3):528-535, 2013.

[29] H. Asorey, D. Cazar-Ramírez, R. Mayo-García, L.A. Núñez, M. Rodríguez-Pascual, L.A. Torres-Niño, and the LAGO Collaboration. Data accessibility, reproducibility and trustworthiness with lago data repository. In The 34th International Cosmic Ray Conference, volume PoS(ICRC2015), page 672, 2015.

[30] R. Barbera, B. Becker, C. Carrubba, G. Inserra, S. Jalife-Villalón, C. Kanellopoulos, K. Koumantaros, R. Mayo-García, L.A. Núñez, O. Prnjate, R. Ricceri, M. Rodríguez-Pascual, A. Rubio-Montero, F. Ruggieri, and CHAIN-REDS Project. A chain-reds solution for accessing computational services. In Actas Cuarta Conferencia de Directores de Tecnología de Información, Gestión de las TICs para la Investigación y la Colaboración, volume TICAL2014, pages 15-24, 2014.

[31] R. Barbera, B. Becker, C. Carrubba, G. Inserra, S. Jalife-Villalón, C. Kanellopoulos, K. Koumantaros, R. Mayo-García, L.A. Núñez, O. Prnjate, R. Ricceri, M. Rodríguez-Pascual, A. Rubio-Montero, F. Ruggieri, and CHAIN-REDS Project. Chain-reds dart challenge. In ANAIS DAS SESSÕES TEMÁTICAS E PÔSTERS, page 166, 2014. 
[32] Y.L. Simmhan, B. Plale, and D. Gannon. A survey of data provenance in e-science. ACM Sigmod Record, 34(3):31-36, 2005.

[33] J. Cooper, J. O. Vik, and D. Waltemath. A call for virtual experiments: accelerating the scientific process. Progress in biophysics and molecular biology, 117(1):99-106, 2015.

[34] R.D. Peng. Reproducible research in computational science. Science (New York, Ny), 334(6060):1226, 2011.

[35] J. Hakala. Persistent identifiers: an overview. KIM Technology Watch Report, 2010.

[36] A. Watson. What next for Ultra High Energy Cosmic Rays?, 2014.

[37] H. Asorey and L.A. Núñez. The pas (polo de astronomia social) project. In Revista Mexicana de Astronomia y Astrofisica Conference Series, volume 44, pages 107-107, 2014.

[38] P. Billoir. A sampling procedure to regenerate particles in a ground detector from a ?thinned? air shower simulation output. Astroparticle Physics, 30(5):270-285, 2008.

[39] B.T. Stokes, R. Cady, D. Ivanov, J.N. Matthews, and G.B. Thomson. Dethinning extensive air shower simulations. Astroparticle Physics, 35(11):759-766, 2012.

[40] A. Estupiñan, H. Asorey, and L. A. Núñez. Implementing the dethinning method for high energy cosmic rays extensive air shower simulations. arXiv preprint, page arXiv:1503.07960, 2015. 